\title{
User Relay assisted Traffic Shifting in LTE-Advanced Systems
}

\author{
Lexi Xu ${ }^{1}$, Yue Chen ${ }^{1}$, KoK Keong Chai ${ }^{1}$, Dantong Liu ${ }^{1}$, Shaoshi Yang ${ }^{2}$, John Schormans ${ }^{1}$, Laurie Cuthbert ${ }^{1}$ \\ ${ }^{1}$ School of Electronic Engineering and Computer Science, Queen Mary University of London, London, United Kingdom \\ ${ }^{2}$ School of Electronics and Computer Science, University of Southampton, Southampton, United Kingdom \\ lexi.xu@eecs.qmul.ac.uk
}

\begin{abstract}
In order to deal with uneven load distribution, mobility load balancing adjusts the handover region to shift edge users from a hot-spot cell to the less-loaded neighbouring cells. However, shifted users suffer the reduced signal power from neighbouring cells, which may result in link quality degradation. This paper employs a user relaying model and proposes a user relay assisted traffic shifting (URTS) scheme to deal with the above problem. In URTS, a shifted user selects a suitable nonactive user as relay user to forward data, thus enhancing the link quality of the shifted user. Since the user relaying model consumes relay user's energy, a utility function is designed in relay selection to reach a trade-off between the shifted user's link quality improvement and the relay user's energy consumption. Simulation results show that URTS scheme could improve SINR and throughput of shifted users. Also, URTS scheme keeps the cost of relay user's energy consumption at an acceptable level.
\end{abstract}

Keywords: mobility load balancing; link quality; relay selection

\section{INTRODUCTION}

Due to service development and user mobility, LTE/LTEAdvanced systems have the random, time-varying and often uneven traffic distribution [1][2]. Mobility load balancing (MLB) is an important resource management functionality that aims at balancing the traffic demand between the hot-spot cell and lightly loaded cells to avoid possible congestion and to increase the spectrum efficiency [3].

Generally, MLB schemes follow two stages: initially, a hotspot cell chooses some less-loaded neighbouring cells as partners; then the hot-spot cell calculates the required offloading traffic and adjusts cell-specific handover offsets $\left(H O_{\text {off }}\right)$ to shift edge users to selected partners. These two stages are designed in our previous work in [4] and [5].

MLB could achieve balanced load distribution. However, shifted users may receive low reference signal received power (RSRP) and suffer link quality degradation. As shown in Fig.1, Cell $l_{1}$ is the hot-spot and tries to offload traffic to the lightly loaded $\mathrm{Cell}_{2}$. $B S_{1}$ increases $\mathrm{HO}_{\text {off }}$ towards $B S_{2}$, in order to trigger handover of $\mathrm{Cell}_{1}$ edge user. Fig.1 clearly shows that after MLB the shifted user receives the reduced $R S R P_{2}$, which is lower than $R S R P_{1}$ before MLB. Furthermore, the reduced RSRP may result in low SINR. In this paper, the phenomenon of the reduced RSRP and even reduced SINR of shifted users is called link quality degradation. This problem impacts networks performance. The shifted user may experience handover failure due to poor link quality. Furthermore, after successful handover, $\mathrm{BS}_{2}$ needs assign more subcarriers to meet the shifted user's data rate requirement, which will reduce the spectrum utilisation.


Fig.1. RSRP comparison of shifted user

To deal with link quality degradation, this paper employs a user relaying model: a non-active user is treated as a relay to forward information to a shifted user. The spatially independent transmission paths (relay link, BS direct link) can achieve spatial diversity, enhancing the shifted user's link quality.

The user relaying model enhances the shifted user's link quality at the expense of relay user's energy consumption. Hence, this paper further proposes a user relay assisted traffic shifting (URTS) scheme. It this scheme, a utility function considering above two factors is designed, which selects an appropriate relay user, enhancing the shifted user's link quality under low cost of relay user's energy consumption.

This paper is organized as follows: Section II presents the user relaying model. Section III analyses this model. Section IV describes the URTS scheme. Simulation results and conclusions are presented in Section V and VI, respectively.

\section{USER RELAYING MODEL}

This work follows our previous research on MLB [4][5]. After MLB implementation, the hot-spot cell offloads its edge users to lightly loaded neighbouring cells. These shifted users may suffer their link quality degradation (see Fig.1).

Meanwhile, there are many non-active users (e.g., sleep mode) in each neighbouring cell [1]. In the downlink of each non-active user, the control channel is partially used while the data channel is idle. Hence, the idle data channel can be utilised to forward transmission data to the shifted user. 
Hence, this paper employs a user relaying model. As shown in Fig.2, the shifted user selects a non-active user located in the lightly loaded cell (which is the handover target cell of the shifted user) as the relay user. When BS transmits data to the shifted user, the relay user receives these data in the first time slot and then forwards to the shifted user in the second time slot.

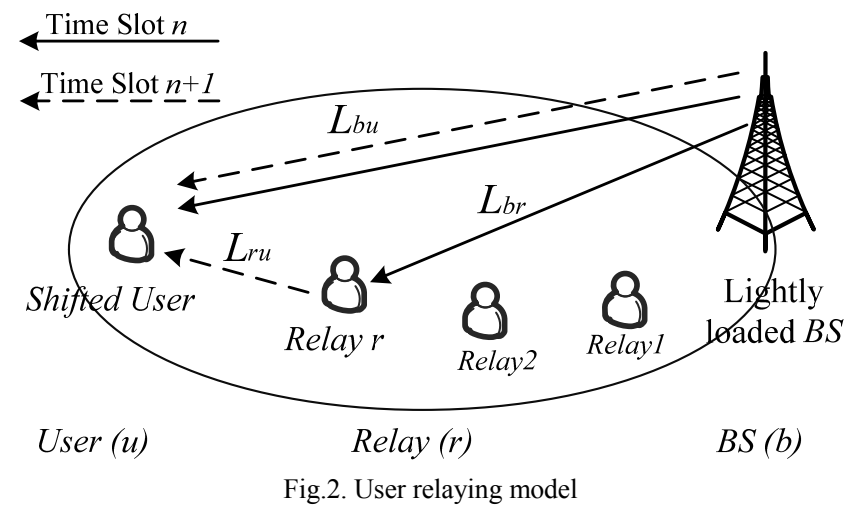

In order to simplify the description, this paper describes the user relaying model as: a destination shifted user, defined as User $u$; several non-active users, defined as Relay $r$ r $\in\{1 \ldots R\}$; and a lightly loaded BS, defined as $B S b$. Therefore, for a specific user relaying model, it consists of one Relay $r$, one shifted $u$ ser $u$ and one source $B S b$.

The downlink transmission mode is shown in Fig.2, including two consecutive time slots [2] [6]. In time slot (TS) $n$, both User $u$ and Relay $r$ listen to the transmission of $B S b$; in TS $n+1$, both BS $b$ and Relay $r$ transmit to User $u$ simultaneously [6]. Note that we assume $B S b$ transmits the same data at two time slots [2] [6].

In this paper, Relay $r$ is operating in the amplified-andforward (AF) mode [7]. In the AF mode, the relay user amplifies all received signals, including interference, noise and user signal. Then it forwards these signals to the shifted user. The AF mode suits the user device, as the AF mode requires lower data processing capability than the decode-andforward (DF) mode does. The parameters that will be used in subsequent sections are listed in Table I:

Table I Parameters of user relaying model

$y_{b r}$ : Relay $r$ 's received signal from $B S b$.

$y_{b u}$ : User $u$ 's received signal, in $B S b$ to User $u$ link.

$y_{r u}$ : User $u$ 's received signal, in Relay $r$ to User $u$ link.

$a_{b r}$ : Channel gain from $B S b$ to Relay $r$.

$a_{b u}$ : Channel gain from $B S b$ to User $u$.

$a_{r u}$ : Channel gain from Relay $r$ to User $u$.

$\lambda_{r}$ : Amplified factor of Relay $r$.

$P_{b}$ : Transmission power of $B S b$.

$P_{r}$ : Transmission power of Relay $r$.

$I_{r}[n]$ : Inter-cell interference at Relay $r$ in TS $n$.

$I_{u}[n]$ : Inter-cell interference at User $u$ in TS $n$.

$C_{u}^{A F r}$ : Throughput of User $u$ with Relay $r$ assistance.

$C_{u}^{N O A F}$ : Throughput of User $u$ without relay assistance.

$C_{r}^{B S}$ : Relay $r$ 's throughput, with the same number of subcarriers

being allocated to Relay $r . C_{r}^{B S}$ reflects Relay $r$ 's throughput loss.

$x_{b}$ : User signal from $B S b$. $\sigma^{2}$ : Common variance of the Gaussian white noise.

$B$ : Bandwidth in the user relaying model.

\section{ANALYSIS OF USER RELAYING MODEL}

Based on the user relaying model, Section III analyses the throughput of shifted User $u$, as well as the impact of energy consumption of Relay $r$.

From the user relaying model, the received signals at User $u$ and Relay $r$ in TS $n$ are given by (1) and (2), respectively.

$$
\begin{aligned}
& y_{b u}[n]=a_{b u} x_{b}[n]+Z_{u}[n]+I_{u}[n] \\
& y_{b r}[n]=a_{b r} x_{b}[n]+Z_{r}[n]+I_{r}[n]
\end{aligned}
$$

where $Z_{u}[n]$ and $Z_{r}[n]$ are the noise at User $u$ and Relay $r$, respectively; $I_{u}[n]$ and $I_{r}[n]$ are the inter-cell interference at User $u$ and Relay $r$, respectively; $a_{b u}$ is the channel gain from $B S b$ to the User $u ; a_{b r}$ is the channel gain from $B S b$ to Relay $r$.

\section{A. SINR of User $u$ in User Relaying Model}

In AF mode [7], Relay $r$ amplifies all received signals and forwards to the shifted User $u$ in TS $n+1$. According to (2), the amplified factor of Relay $r$ can be expressed as $\lambda_{r}$, using (3):

$$
\lambda_{r}^{2}\left(\left|a_{b r}\right|^{2} P_{b}+\sigma^{2}+\left|I_{r}[n]\right|^{2}\right)=P_{r} \Rightarrow \lambda_{r}^{2}=\frac{P_{r}}{\left|a_{b r}\right|^{2} P_{b}+\sigma^{2}+\left|I_{r}[n]\right|^{2}}
$$

where $P_{b}$ and $P_{r}$ are the transmission power of $B S b$ and Relay $r$, respectively; $\sigma^{2}$ is the common variance of the Gaussian white noise; || denotes the magnitude of the symbol. For example, $\left|I_{r}[n]\right|^{2}$ is the interference power at Relay $r$.

In TS $n+1$, User $u$ received signals from Relay $r$ and $B S b$ are discussed in $i$ ) and ii).

i) User $u$ received signal from Relay $r$ (Link $L_{r u}$ in Fig.2) in TS $n+1$ is denoted as $y_{r u}[n+1]$, using (4):

$$
\begin{aligned}
& y_{r u}[n+1]=\lambda_{r} y_{b r}[n] a_{r u}+Z_{u}[n+1]+I_{u}[n+1] \\
& =\left(\lambda_{r} a_{r u} a_{b r} x_{b}[n]\right)+\left(\lambda_{r} a_{r u} Z_{r}[n]+Z_{u}[n+1]\right)+\lambda_{r} a_{r u} I_{r}[n]+I_{u}[n+1]
\end{aligned}
$$

where $y_{b r}[n]$ refers to (2); $a_{r u}$ is the channel gain from Relay $r$ to User $u$. According to (4), SINR of User $u$ in TS $n+1$ from $L_{r u}$ can be expressed as $S I N R_{u, n+1}^{\left(L_{r u}\right)}$, using (5):

$$
\operatorname{SINR}_{u, n+1}^{\left(L_{r u}\right)}=\frac{P_{b}\left|a_{r u}\right|^{2}\left|a_{b r}\right|^{2} \lambda_{r}^{2}}{\left|a_{r u}\right|^{2} \lambda_{r}^{2}\left(\left|I_{r}[n]\right|^{2}+\sigma^{2}\right)+\left|I_{u}[n+1]\right|^{2}+\sigma^{2}}
$$

ii) User $u$ received signal from $B S b$ (Link $L_{b u}$ in Fig.2) in TS $n+1$ is denoted as $y_{b u}[n+1]$, using (6). From (6), SINR of User $u$ in TS $n+1$ from $L_{b u}$ is denoted as $\operatorname{SINR}\left(R_{u, n+1}^{\left(L_{b u}\right)}\right.$, using (7):

$$
\begin{gathered}
y_{b u}[n+1]=a_{b u} x_{b}[n+1]+Z_{u}[n+1]+I_{u}[n+1] \\
\operatorname{SINR}_{u, n+1}^{\left(L_{b u}\right)}=\frac{P_{b}\left|a_{b u}\right|^{2}}{\left|I_{u}[n+1]\right|^{2}+\sigma^{2}}
\end{gathered}
$$


iii) Besides, in TS $n$, User $u$ received signal from $B S b$ (Link $L_{b u}$ in Fig.2) is shown in (1). Hence, SINR of User $u$ in TS $n$ from $L_{b u}$ can be expressed as $S I N R_{u, n}^{\left(L_{b u}\right)}$, using (8):

$$
\operatorname{SINR}_{u, n}^{\left(L_{b u}\right)}=\frac{P_{b}\left|a_{b u}\right|^{2}}{\left|I_{u}[n]\right|^{2}+\sigma^{2}}
$$

In (1) (4) (6), $x_{b}[n]$ and $x_{b}[n+1]$ are the same user signal from three separate links. User $u$ combines them to enhance the signal quality. According to [6], the SINR achieved after signal processing at $U \operatorname{ser} u$ is defined as $\operatorname{SINR}_{u}^{A F r}$, using (9):

$$
\begin{gathered}
\operatorname{SINR}_{u}^{A F r}=\operatorname{SINR}_{u, n}^{\left(L_{b u}\right)}+\operatorname{SINR}_{u, n+1}^{\left(L_{r u}\right)}+\operatorname{SINR}_{u, n+1}^{\left(L_{b u}\right)} \\
=\frac{P_{b}\left|a_{b u}\right|^{2}}{\left|I_{u}[n]\right|^{2}+\sigma^{2}}+\frac{P_{b}\left|a_{r u}\right|^{2}\left|a_{b r}\right|^{2} \lambda_{r}^{2}}{\left|a_{r u}\right|^{2} \lambda_{r}^{2}\left(\left|I_{r}[n]\right|^{2}+\sigma^{2}\right)+\left|I_{u}[n+l]\right|^{2}+\sigma^{2}}+\frac{P_{b}\left|a_{b u}\right|^{2}}{\left|I_{u}[n+1]\right|^{2}+\sigma^{2}}
\end{gathered}
$$

\section{B. Throughput of User $u$ with Relay $r$ assistance}

Based on $\operatorname{SINR}_{u}^{A F r}$, the throughput of User $\boldsymbol{u}$ with Relay $\boldsymbol{r}$ assistance is defines as $C_{u}^{A F r}$, using (10):

$$
\begin{gathered}
C_{u}^{A F r}=\frac{B}{2} \log _{2}\left(1+\operatorname{SINR}_{u}^{A F r}\right) \\
=\frac{B}{2} \log _{2}\left(1+\frac{P_{b}\left|a_{b u}\right|^{2}}{\left|I_{u}[n]\right|^{2}+\sigma^{2}}+\frac{P_{b}\left|a_{r u}\right|^{2}\left|a_{b r}\right|^{2} \lambda_{r}^{2}}{\left|a_{r u}\right|^{2} \lambda_{r}^{2}\left(\left|I_{r}[n]\right|^{2}+\sigma^{2}\right)+\left|I_{u}[n+1]\right|^{2}+\sigma^{2}}+\frac{P_{b}\left|a_{b u}\right|^{2}}{\left|I_{u}[n+I]\right|^{2}+\sigma^{2}}\right)
\end{gathered}
$$

where $B$ is the bandwidth; $\frac{1}{2}$ denotes that User $u$ receives the same transmission data in two time slots [2] [6].

Relay selection impacts the value of $\left|a_{r u}\right|^{2},\left|a_{b r}\right|^{2}$ and $\left|I_{r}[n]\right|^{2}$. From Equation (10), selecting a suitable relay user could improve the throughput of the shifted user.

\section{Throughput of User $u$ without relay}

If there isn't a relay link, User $u$ only receives a signal from $B S b$ (Link $L_{b u}$ in Fig.2) in TS $n$ and TS $n+1$. From (7) (8), the throughput of $\boldsymbol{U}$ ser $\boldsymbol{u}$ without relay is defined as $C_{u}^{N O A F}$ :

$$
C_{u}^{N O A F}=\frac{B}{2} \log _{2}\left\{1+\frac{P_{b}\left|a_{b u}\right|^{2}}{\left|I_{u}[n]\right|^{2}+\sigma^{2}}+\frac{P_{b}\left|a_{b u}\right|^{2}}{\left|I_{u}[n+l]\right|^{2}+\sigma^{2}}\right\}
$$

\section{Throughput Loss of Relay $r$}

From the user relaying model, Relay $r$ amplifies signal power and forwards to User $u$ in TS $n+1$. This consumes the energy of Relay $r$ and shortens Relay $r$ battery working time, which will result in the throughput loss of Relay $r$. This paper uses throughput as the single metric, which allows us to compare the benefit to shifted users and the cost to relays directly. We define $C_{r}^{B S}$ as Relay r's throughput, with the same number of subcarriers (the same bandwidth) being allocated to Relay $r$. Hence, $C_{r}^{B S}$ reflects Relay r's throughput loss, and indicates the impact of energy consumption of Relay $r$.

If Relay $r$ becomes active, the received signal at Relay $r$ in TS $n+1$ is given by (12). Correspondingly, the achieved SINR of Relay $r$ in TS $n+1$ is defined as $\operatorname{SINR}_{r, n+1}$, using (13).

$$
y_{b r}[n+1]=a_{b r} x_{b}[n+1]+Z_{r}[n+1]+I_{r}[n+1]
$$

$$
\operatorname{SINR}_{r, n+1}=\frac{P_{b}\left|a_{b r}\right|^{2}}{\left|I_{r}[n+1]\right|^{2}+\sigma^{2}}
$$

where $P_{b}$ is the transmission power of $B S b ;\left|I_{r}[n+1]\right|^{2}$ is the interference power at Relay $r$ in TS $n+1 . a_{b r}$ is the channel gain from $B S b$ to Relay $r$. According to (13), $C_{r}^{B S}$ can be expressed as (14):

$$
C_{r}^{B S}=\frac{B}{2} \log _{2}\left\{1+\frac{P_{b}\left|a_{b r}\right|^{2}}{\left|I_{r}[n+l]\right|^{2}+\sigma^{2}}\right\}
$$

Relay selection impacts the value of $\left|a_{b r}\right|^{2}$ and $\left|I_{r}[n+1]\right|^{2}$. Equation (14) indicates that selecting an appropriate relay could keep the throughput loss of the relay user at a low level.

\section{User Relay Assisted Traffic ShIFGING SCHEME}

From the analysis above, the user relaying model provides a complementary link to improve the throughput of the shifted user. However, this model also consumes the battery power of the relay user and shortens its working time, which will reduce the relay user's total throughput. Both the factor of the shifted user's throughput and the factor of the relay user's throughput loss should be considered jointly in relay selection.

Therefore, based on the user relaying model, this paper proposes a user relay assisted traffic shifting (URTS) scheme. The key of URTS scheme lies in designing a utility function to select an appropriate relay for the two factors' trade-off.

\section{A. Weight of Traffic Shifting}

In order to select a proper Relayr to increase the throughput of the shifted User $u$, this paper designs the weight of traffic shifting (WTS) as $\Psi_{r, W T S}$. As shown in (15), $\Psi_{r, W T S}$ equals the ratio of User $u$ 's throughput with Relay $r$ assistance $\left(C_{u}^{A F r}\right.$, see (10)) to User $u$ 's throughput without relay $\left(C_{u}^{N O A F}\right.$, see (11)). Hence, $\Psi_{r, W T S}$ indicates the throughput gain of User $u$.

$$
\psi_{r, W T S}=\frac{C_{u}^{A F r}}{C_{u}^{N O A F}} \quad r \in\{1,2 \ldots R\}
$$

\section{B. Weight of Throughput Loss}

The energy consumption of Relay $r$ will shorten its battery working time and reduce its total throughput. Under the similar energy consumption of the non-active Relay $r$, this paper designs the weight of throughput loss (WTL) to compare the throughput loss of Relay $r$, and the throughput improvement for User $u$. $\Psi_{r, W T L}$ is calculated as (16):

$$
\psi_{r, W T L}=\frac{C_{r}^{B S}}{C_{u}^{A F r}-C_{u}^{N O A F}} \quad r \in\{1,2 \ldots R\}
$$

* $C_{u}^{A F r}-C_{u}^{N O A F}$ reflects the throughput improvement of User $u$, with Relay $r$ assistance.

${ }^{*} C_{r}^{B S}$ reflects Relay r's throughput loss itself (see (14)).

$\Psi_{r, W T L}$ indicates the impact of energy consumption. In (16), the higher throughput loss of Relay $r$ leads to the higher $\Psi_{r, W T L}$. 


\section{Utility Function based Relay Selection}

In order to select a suitable user to reach the trade-off between the weight of traffic shifting and the weight of throughput loss, this paper designs a utility function $\mathcal{u}_{r}$ as (17):

$$
u_{r}=\frac{\psi_{r, W T S}}{\psi_{r, W T L}}=\frac{C_{u}^{A F r} \times\left(C_{u}^{A F r}-C_{u}^{N O A F}\right)}{C_{u}^{N O A F} \times C_{r}^{B S}} \quad r \in\{1 \ldots R\}
$$

According to (17), the higher User $u$ 's throughput with Relay $r$ assistance could lead to higher $u_{r}$. Meanwhile, the lower throughput loss of Relay $r$ could lead to higher $u_{r}$. Hence, URTS scheme tries select Relay $k$ to maximize $u_{r}$ :

$$
\text { Relay } k=\underset{r \in\{1,2 \ldots R\}}{\operatorname{argmax}} u_{r}=\underset{r \in\{1,2 \ldots R\}}{\operatorname{argmax}} \frac{C_{u}^{A F r} \times\left(C_{u}^{A F r}-C_{u}^{N O A F}\right)}{C_{u}^{N O A F} \times C_{r}^{B S}}
$$

From (18), the utility function relates to $C_{u}^{A F r}, C_{r}^{B S}, C_{u}^{N O A F}$. User $u$ has its correspondingly fixed $C_{u}^{N O A F}$, given by (11).

$C_{u}^{A F r}$ and $C_{r}^{B S}$ are varying with different Relay $r$. From (10), $C_{u}^{A F r}$ is based on three varying parameters: $\left|a_{r u}\right|^{2},\left|a_{b r}\right|^{2}$, $\left|I_{r}[n]\right|^{2}$. From (14), $C_{r}^{B S}$ is based on $\left|a_{b r}\right|^{2}$ and $\left|I_{r}[n+1]\right|^{2}$.

\section{URTS Scheme Process}

Based on the analysis above, User $u$ could calculate the utility function to select suitable relay, only under knowing the value of $\left|a_{b r}\right|^{2},\left|a_{r u}\right|^{2},\left|I_{r}[n]\right|^{2},\left|I_{r}[n+1]\right|^{2}$. To reduce the complexity and signalling load, the URTS scheme calculates them according to existing/measurable parameters in other resource management functionalities, e.g., cell selection, admission control. Specifically, they can be estimated as:

i) $\left|a_{b r}\right|^{2}$ ( $a_{b r}$ is the channel gain from $B S b$ to Relay $r$ ): Since Relay $r$ knows its received RSRP from $B S b$, as well as $B S b$ 's transmission power ( which could be informed from $B S$ $b$ in control channel [1]), Relay $r$ estimates $\left|a_{b r}\right|^{2}$ as (19):

$$
\left|a_{b r}\right|^{2} \approx \frac{\text { Relay } r \text { 's received } R S R P \text { from } B S b}{B S \text { b's transmission power } P_{b}}
$$

ii) $\left|a_{r u}\right|^{2}$ ( $a_{r u}$ is the channel gain from Relay $r$ to User $u$ ): After Relay $r$ responding to User $u$, User $u$ knows its received response signal power from Relay $r$. Besides, Relay $r$ reports $P_{r}$ (see Fig.3) to User $u$. User $u$ calculates $\left|a_{r u}\right|^{2}$ as (20):

$$
\left|a_{r u}\right|^{2} \approx \frac{\text { User } u \text { 's received response power from Relay } r}{\text { Relay } r \text { 's transmission power } P_{r}}
$$

iii) $\left|I_{r}[n]\right|^{2},\left|I_{r}[n+1]\right|^{2}$ (interference power of Relay $r$ in TS $n$ and $n+1$ ): In the full frequency reuse LTE-Advanced cellular networks [1] [4], precise interference estimation is difficult. It is because Relay r's interference, which is imposed by other cells using the co-channel subcarriers, is varying due to the dynamic subcarriers allocation of neighbouring cells. To reduce the estimation complexity, Relay $r$ considers the RSRP from all neighbouring BSs as the interference, and then calculates the theoretically heaviest interference $\left|I_{r}\right|^{2}$.

The flowchart of URTS scheme is shown in Fig.3, which involves the process of shifted User $u$ and Relay $r$.



Fig.3. Flowchart of URTS scheme

As shown in Fig.3, if a user in the hot-spot BS needs to be shifted to the target $B S b$, the shifted User $u$ broadcasts its cooperation request and its target $B S I D$ (denotes $B S b$ ).

After receiving the broadcast, the non-active user judges whether it is in the coverage of $B S b$ and whether it is available to assist User $u$ (Since a non-active user can only assist a shifted user at a time). If it is, this non-active Relay $r$ calculates $\left|a_{b r}\right|^{2}$ from (19). Besides, Relay $r$ estimates $\left|I_{r}\right|^{2}$ as the sum of RSRP from all neighbouring BSs. Then Relay $r$ responds and sends $\left|a_{b r}\right|^{2},\left|I_{r}\right|^{2}$, and $P_{r}$ to User $u$.

After receiving the responses, User $u$ calculates $\left|a_{r u}\right|^{2}$ from (20). User $u$ calculates the utility function $u_{r}$ of all responding non-active users, based on $\left|a_{r u}\right|^{2},\left|a_{r u}\right|^{2}, C_{u}^{N O A F}$ (User $u$ calculates its correspondingly $C_{u}^{N O A F}$ from (11)). Then User $u$ selects a non-active user with the largest $u_{r}$ as relay.

After relay user selection, the selected non-active user starts to assist User $u$ to forward the transmission data.

Note that: multiple shifted users may request one non-active user at the same time. Under this scenario, the non-active user chooses one shifted user, from which the non-active user receives the strongest broadcast power. It is because the high received power indicates good link quality between two users.

\section{Simulation Analysis}

\section{A. Simulation Scheme Introduction}

This paper designs a downlink system-level LTE-Advanced simulation platform based on [1]. As shown in Fig.4, there are three hot-spot areas, which cover $70 \%$ active users and $70 \%$ non-active users. Other important parameters are shown in Table II. Besides, this paper simulates four schemes.

Table II Simulation Platform Parameters

\begin{tabular}{|c|c|}
\hline Parameter & Value \\
\hline Subcarrier and Total bandwidth & Subcarrier: $15 \mathrm{~K} \mathrm{~Hz}$; Total: $5 \mathrm{M} \mathrm{Hz}$ \\
\hline Physical resource blocks(PRB) & Total 25 (12 subcarriers per PRB) \\
\hline
\end{tabular}




\begin{tabular}{|c|c|}
\hline Frequency & $2 \mathrm{G} \mathrm{Hz}$ \\
\hline Inter-site distance & $500 \mathrm{~m}$ (3-sector antenna splits site) \\
\hline Log-normal shadow fading & Standard Deviation: $8 \mathrm{~dB}$ \\
\hline BS-MS Path loss model & $37.6 \lg (r)+128.1, r-k m$ \\
\hline MS-MS Path loss model & $-27.6+20 \lg (2000)+20 \lg (\mathrm{d})$, d-meter $[8]$ \\
\hline Total BS transmission power & $43 \mathrm{dBm}$ \\
\hline Total MS transmission power & $21 \mathrm{dBm}$ \\
\hline User mobility & Speed: $3 \mathrm{~m} / \mathrm{s}$; Direction: Random \\
\hline Scheduler & Max C/I \\
\hline Traffic model & Guaranteed 64kbps \\
\hline
\end{tabular}

gradually adjusts cell-specific $H O_{\text {off }}$ towards each partner to offload users until two cells reach a similar load.

\section{iv) WTS based user relay assisted traffic shifting scheme (named CLB with WTS user relay in Fig.6-9)}

Section $\mathrm{V}$ tries to evaluate whether the proposed utility function could improve the performance of shifted users under acceptable throughput loss of relay users. Therefore, the reference $C L B$ with WTS user relay scheme is simulated. Its simulation flow is similar to Fig.5. The only difference is that a shifted user only considers the proposed WTS (weight of traffic shifting) $\Psi_{r, W T S}$ during the relay selection. As discussed in Section IV A, CLB with WTS user relay scheme aims at selecting the relay which can best improve the throughput of the shifted user, while it does not consider the throughput loss of relay users.

\section{B. Simulation Results}

Load balancing (LB) handover failure rate reflects the link quality of shifted users [1], because the better the link provided by a partner cell, the more shifted users can be handed over successfully. Fig.6 shows that the CLB scheme has lower LB handover rate than the typical $M L B$ scheme. Compared with the $C L B$ scheme, the proposed $C L B$ with utility function user relay scheme can further reduce the LB handover failure rate. It is because the relay link could enhance the link quality of the shifted user. As a result, the improved link quality decreases the LB handover failure rate.

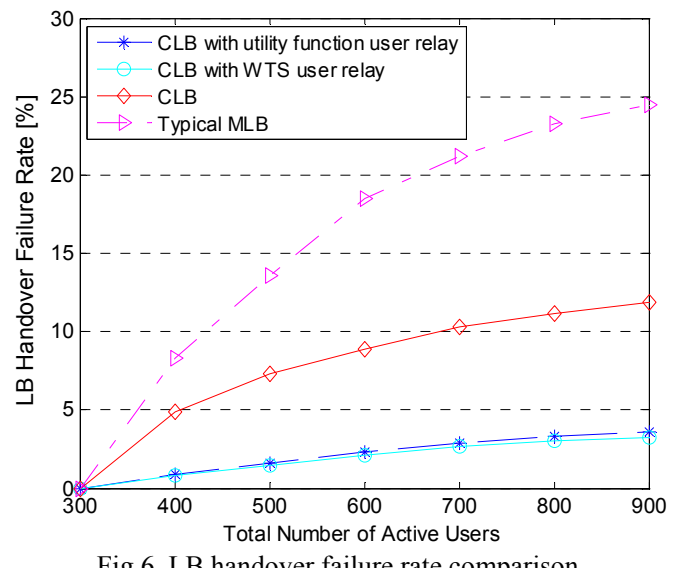

Fig.6. LB handover failure rate comparison previous cooperative load balancing (CLB) scheme, consisting of user-vote assisted partner selection [4] and cooperative traffic shifting [5]. After CLB, the shifted user triggers the proposed utility function based relay selection. Finally, the relay user assists the transmission of the shifted user.

\section{ii) CLB scheme}

This work also simulates the standalone $C L B$ scheme (without user relay) [4][5]. In the $C L B$ scheme, the hot-spot cell adjusts $H O_{\text {off }}$ towards partner Cell $_{b}$. Then User $u$ in the hot-spot cell will be shifted to $\mathrm{Cell}_{b}$ without relay assistance.

\section{iii) Typical MLB scheme}

In addition, the typical mobility load balancing (MLB) scheme [3] in LTE is simulated for comparison. In [3], the hot-spot cell selects all less-loaded neighbouring cells as partners, then the hot-spot cell estimates its shifted users' required subcarriers in each partner cell. The hot-spot cell

In order to evaluate the proposed $C L B$ with utility function user relay scheme in helping shifted users of different link qualities, Fig.7 divides shifted users into five categories, according to their SINR after shifting (without user relay assistance): SINR lower than 1; between 1 and 2 ; between 2 and 6 ; between 6 and 12; between 12 and 18 .

Among five categories, the poor link quality shifted users $(\mathrm{SINR}<1)$, experience the largest SINR improvement via the proposed $C L B$ with utility function user relay scheme. For example, Fig.7 shows that the proposed scheme can increase up to $110 \%$ SINR for shifted users in SINR $<1$ category, as well as increase $75 \%$ for shifted users in $1<$ SINR $<2$ category.

The proposed scheme also effectively improves SINR for the medium link quality shifted users. For example, the shifted users' SINR can be improved up to $32 \%$ and $20 \%$ in $2<$ SINR $<6$ category and $6<$ SINR $<12$ category, respectively. 
Fig.7 also shows that the proposed scheme could slightly increase the SINR of good link quality shifted users, e.g., $12<$ SINR $<18$ category. But their SINR enhancements are not as outstanding as the poor/medium link quality users.

From the analysis above, the proposed scheme is more useful for the shifted users who suffer poor link quality. Due to the improved SINR and the reduced handover failure rate, Fig.8 shows that the CLB with utility function user relay scheme can improve the overall throughput of all shifted users, compared to $C L B$ scheme.

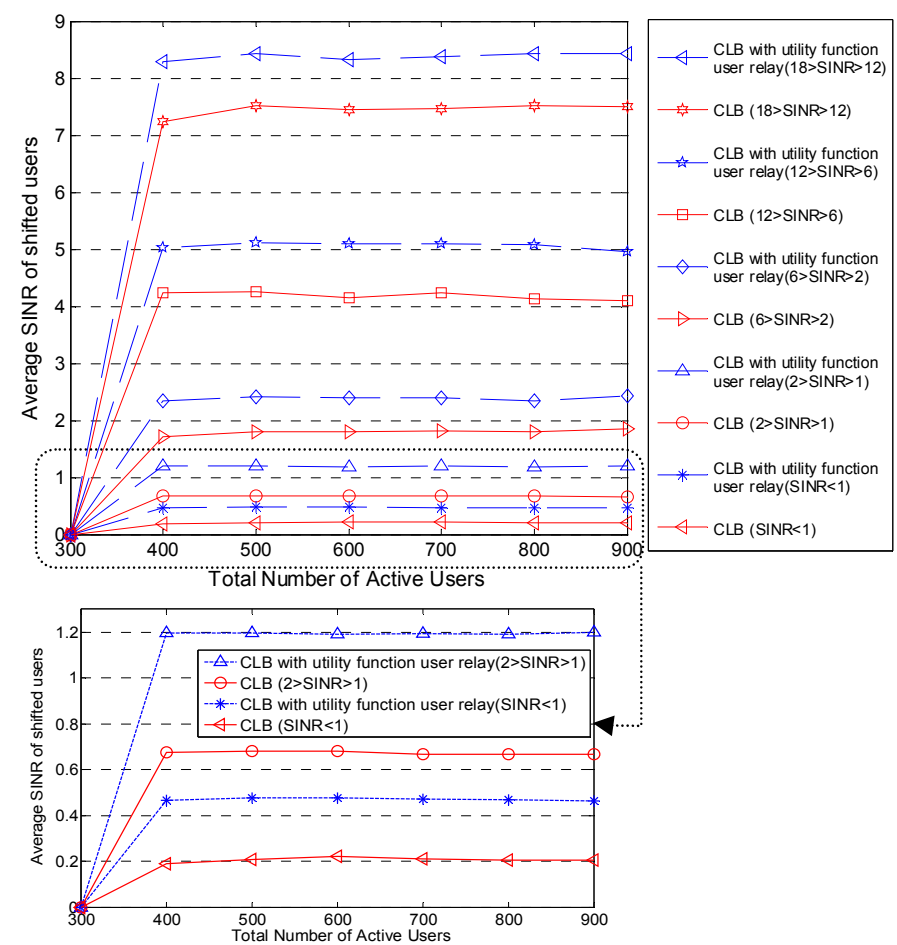

Fig.7 SINR comparison of shifted users in different SINR categories



Fig.8. Overall throughput of all shifted users

Fig.6, 8, 9 further evaluate the utility function in the trade-off between shifted users performance and relay users performance.

In Fig.8, both the CLB with utility function user relay scheme and $C L B$ with WTS user relay scheme can improve the overall throughput of shifted users. For example, under 700 users scenario, the CLB with utility function user relay scheme can increase the throughput by $31 \%$ and the CLB with WTS user relay scheme can increase the throughput by $35 \%$, compared to the CLB scheme. The reason for the slight difference is that the WTS scheme only aims at selecting the relay user to most improve the throughput of the shifted user, while the utility function also considers the throughput loss of the relay user. Besides, Fig.6 clearly shows that CLB with utility function user relay scheme has a similar LB handover failure rate as the $C L B$ with WTS user relay scheme.

Fig.9 shows the overall throughput loss of relay users. The throughput loss in the CLB with utility function user relay scheme is $23 \% \sim 30 \%$ less than that in the CLB with WTS user relay scheme.

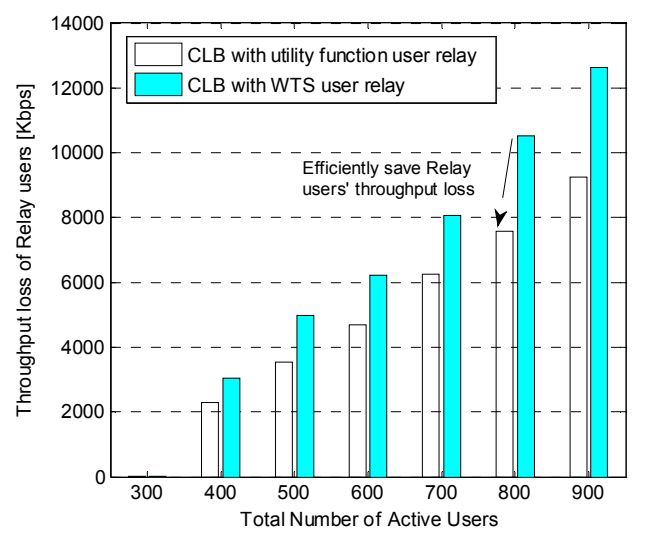

Fig.9 Overall throughput loss of all relay users

In summary, Fig.6, 8, 9 verifies that shifted users have similar performance in CLB with utility function user relay scheme and CLB with WTS user relay scheme. Meanwhile, $C L B$ with utility function user relay scheme can significantly reduce the throughput loss of relay users. Therefore, the proposed utility function can reach the trade-off between shifted users' performance and relay users' throughput loss.

\section{CONCLUSION}

This paper employs a user relaying model to enhance the link quality of shifted users in load balancing. Furthermore, based on this model, a user relay assisted traffic shifting (URTS) scheme is proposed. URTS scheme could effectively increase the link quality of shifted users under accepted cost of relay users' energy consumption.

\section{REFERENCES}

[1] 3GPP TS 36.300 V9.5.0, "E-UTRAN Overall description," Sept. 2010

[2] Liping Wang, Yusheng Ji, Fuqiang Liu, "Resource allocation for OFDMA relay-enhanced system with cooperative selection diversity," in Proc. IEEE WCNC, Apr. 2009, Budapest, Hungry

[3] R. Kwan, R. Arnott, R. Paterson, "On mobility load balancing for LTE systems," in Proc. IEEE VTC-fall, Sept. 2010, Ottawa, Canada

[4] Lexi Xu, Yue Chen, John Schormans, Laurie Cuthbert, Tiankui Zhang, "User-vote assisted self-organizing load balancing for OFDMA cellular systems," in Proc. IEEE PIMRC, Sept.2011, Toronto, Canada, pp.217-221

[5] Lexi Xu, Yue Chen, Kok Keong Chai, Tiankui Zhang, John Schormans, Laurie Cuthbert, "Cooperative load balancing for OFDMA cellular networks," in Proc. European Wireless, Apr. 2012, Poznan, Poland

[6] Basak Can, Halim Yanikomeroglu, Furuzan Atay Onat, Elisabeth De Carvalho, Hiroyuki Yomo, "Efficient cooperative diversity schemes and radio resource allocation for IEEE 802.16j," in Proc. IEEE WCNC, Apr. 2008, Las Vagus, USA, pp.36-41

[7] Jun Cai, Xuemin Shen, J. W. Mark, A. S. Alfa, "Semi-distributed user relaying algorithm for amplify-and-forward wireless relay networks," IEEE Trans. Wireless Commun., vol.7, no. 4, Apr. 2008, pp.1348-1357

[8] Zhenyu Wang, E. K. Tameh, A. R. Nix, "Statistical peer-to-peer channel models for outdoor urban environments at $2 \mathrm{GHz}$ and $5 \mathrm{GHz}$," in Proc. IEEE VTC-fall, Sept. 2004, Los Angeles, USA 